Check for updates

Cite this: RSC Adv., 2018, 8, 14462

Received 26th February 2018

Accepted 12th April 2018

DOI: $10.1039 / c 8 r a 01680 a$

rsc.li/rsc-advances

\title{
Coreashell structured Co-CoO@NC nanoparticles supported on nitrogen doped carbon with high catalytic activity for oxygen reduction reaction $\uparrow$
}

\author{
Zihao Zhen, ${ }^{a}$ Zhongqing Jiang, (D) bc Xiaoning Tian, ${ }^{\mathrm{C}}$ Lingshan Zhou, ${ }^{\mathrm{a}}$ Binglu Deng, ${ }^{\mathrm{a}}$ \\ Bohong Chen ${ }^{a}$ and Zhong-Jie Jiang (D) *a
}

\begin{abstract}
A composite with a hierarchical structure consisting of nitrogen doped carbon nanosheets with the deposition of nitrogen doped carbon coated $\mathrm{Co}-\mathrm{CoO}$ nanoparticles (Co-CoO@NC/NC) has been synthesized by a simple procedure involving the drying of the reaction mixture containing $\mathrm{Co}\left(\mathrm{NO}_{3}\right)_{2}$, glucose, and urea and its subsequent calcination. The drying step is found to be necessary to obtain a sample with small and uniformly sized $\mathrm{Co}-\mathrm{CoO}$ nanoparticles. The calcination temperature has a great effect on the catalytic activity of the final product. Specifically, the sample prepared at the calcination temperature of $800{ }^{\circ} \mathrm{C}$ shows better catalytic activity of the oxygen reduction reaction (ORR). Urea in the reaction mixture is crucial to obtain the sample with the uniformly sized Co-CoO nanoparticles and also plays an important role in improving the catalytic activity of the Co-CoO@NC/NC. Additionally, there exists a strong electronic interaction between the Co-CoO nanoparticles and the NC. Most interestingly, the Co-COO@NC/NC is highly efficient for the ORR and can deliver an ORR onset potential of $0.961 \mathrm{~V}$ vs. RHE and a half-wave potential of $0.868 \mathrm{~V}$ vs. RHE. Both the onset and half-wave potentials are higher than those of most catalysts reported previously and even close to those of the commercial Pt/C (the ORR onset and half-wave potential of the Pt/C are 0.962 and $0.861 \mathrm{~V} v$ s. RHE, respectively). This, together with its high stability, strongly suggests that the Co-CoO@NC/NC could be used as an efficient catalyst for the ORR.
\end{abstract}

\section{Introduction}

The ever-increasing global demands in sustainable energy have prompted worldwide efforts in developing alternative energy conversion and storage devices, such as fuel cells, and metal air batteries. ${ }^{\mathbf{1} 2}$ Exploiting efficient electrocatalysts that can enhance the kinetics of the oxygen reduction reaction (ORR) has therefore attracted a great deal of attention, since the sluggishness of the ORR has greatly limited practical applications of these devices. ${ }^{3,4}$ Currently, the best catalysts reported for the ORR are $\mathrm{Pt}$ and its alloys, such as $\mathrm{Pt} / \mathrm{Ni}, \mathrm{Pt} / \mathrm{Co}$, and $\mathrm{Pt} / \mathrm{Cu}$, etc. ${ }^{5-8}$ These catalysts, however, suffer from severe problems of low

${ }^{a}$ Guangzhou Key Laboratory for Surface Chemistry of Energy Materials, New Energy Research Institute, College of Environment and Energy, South China University of Technology, Guangzhou 510006, Guangdong, P. R. China. E-mail: Zhongjiejiang1978@hotmail.com; eszjiang@scut.edu.cn

${ }^{b}$ Department of Physics, Key Laboratory of ATMMT Ministry of Education, Zhejiang SciTech University, Hangzhou 310018, P. R. China

${ }^{c}$ School of Materials and Chemical Engineering, Ningbo University of Technology, Ningbo 315211, Zhejiang, P. R. China

$\dagger$ Electronic supplementary information (ESI) available: Element mapping; TGA curve; TEM images; XPS survey spectra; XRD patterns; Raman spectra; LSVs and K-L plots; elemental composition; performance comparison. See DOI: 10.1039/c8ra01680a stability, poor tolerance to poisoning, scarcity in earth, and high cost, which have largely hindered their applications as the electrocatalysts for the ORR in fuel cells and metal air batteries. ${ }^{9,10}$ Developing alternative catalysts that can boost the ORR kinetics and simultaneously meet the requirements of low cost and high stability is therefore highly desirable.

In response to reduce the cost and improve the catalytic activities for the ORR, efforts on developing ORR catalysts have been mainly focused on noble metal-free or even metal-free materials. ${ }^{11,12}$ Transition metal based catalysts are of particular interest due to their advantages of superior catalytic activity and earth abundance. ${ }^{\mathbf{1 3 , 1 4}}$ As reported previously, transition metals (TMs) and their oxides (TMOs) can exhibit excellent catalytic activities for the ORR when they are synthesized properly. ${ }^{15-18}$ The main problem associated with the practical applications of the TMs and TMOs, however, is their poor stability in the complicated electrochemical environments. ${ }^{1920}$ Strategies to address this issue are therefore needed to promote the applications of the TMs and TMOs as the ORR electrocatalysts. Encapsulating with the carbon shell is a promising way to improve the stability of the TMs and TMOs. ${ }^{16,21-23}$ As demonstrated recently, the encapsulation cannot only protect the encapsulated TMs and TMO cores from degradation through avoiding their direct contact with the electrolyte solutions, but also improve the catalytic activities of 
the TMs and TMOs since the ultrathin carbon shells can greatly promote penetration of electrons from the TM and TMO cores to the carbon surface, participating in the catalytic reactions. ${ }^{24,25}$ In particular, when the carbon shell is doped with heteroatoms, the synergistic interaction between heteroatom doped carbon shell and the TM/TMO core can increase the electron density on the carbon shell, further improving their capability for the catalytic applications. ${ }^{24,26,27}$

Metallic cobalt ( $\mathrm{Co})$ and its oxide (CoO) are typical TM based materials, both of which have been demonstrated to be usable as the catalysts for the ORR. ${ }^{28,29}$ The catalytic activities of the single component $\mathrm{Co}$ or $\mathrm{CoO}$ material, however, remain lower in comparison to the Pt-based catalysts, although they might be enhanced through the integration of $\mathrm{Co}$ or $\mathrm{CoO}$ with carbonaceous materials. ${ }^{20,30}$ Recently, the composite materials consisting of two or more components that are individually active for the ORR are receiving growing attention, since the synergistic between multiple components might generate more fascinating properties for the catalytic applications. ${ }^{2,12,31-36}$ For example, the $\mathrm{Fe}-\mathrm{Fe}_{2} \mathrm{O}_{3}$ particles deposited $\mathrm{N}$-doped graphene shows enhanced catalytic activity for the ORR. ${ }^{12}$ The Ni-NiO nanoparticles on PDDA-modified graphene are highly active for oxygen reduction reaction. ${ }^{37}$ In this sense, the combination of Co with $\mathrm{CoO}$ is therefore expectable to produce a composite particle with higher catalytic activity for the ORR.

In this work, we investigate the ORR catalytic activity of nitrogen doped carbon coated $\mathrm{Co}-\mathrm{CoO}$ nanoparticles (CoCoO@NC). Specifically, a composite material with a hierarchical structure consisting of the nitrogen doped carbon nanosheets with the deposition of the $\mathrm{Co}-\mathrm{CoO} @ \mathrm{NC}$ (Co$\mathrm{CoO} @ \mathrm{NC} / \mathrm{NC}$ ) has been synthesized through a simple calcination of a mixture containing $\mathrm{Co}\left(\mathrm{NO}_{3}\right)_{2}$, glucose, and urea. The effects of the drying step and the calcination temperature on the catalytic activity of the final product has been studied. It shows that the drying step is indispensable in obtaining the sample with the small and uniformly sized $\mathrm{Co}-\mathrm{CoO}$ nanoparticles. The sample prepared at the calcination temperature of $800^{\circ} \mathrm{C}$ shows better catalytic activity for the ORR, since at this temperature glucose and urea are well carbonized and the obtained product consists of small and uniform sizes of the $\mathrm{Co}-\mathrm{CoO}$ nanoparticles (NPs) and a high content of the doping nitrogen in the carbon. Urea in the reaction mixture is found to play an important role in the formation of the $\mathrm{Co}-\mathrm{CoO} @ \mathrm{NC} / \mathrm{NC}$ with higher catalytic activity. It does not only provide a nitrogen source for the NC, but also facilitate the formation of the Co$\mathrm{CoO}$ nanoparticles with small and uniform sizes. Most notably, there exists a strong interaction between the $\mathrm{Co}-\mathrm{CoO}$ nanoparticles and the NC. When used as the catalyst, the Co$\mathrm{CoO} @ \mathrm{NC} / \mathrm{NC}$ at the calcination temperature of $800{ }^{\circ} \mathrm{C}(\mathrm{Co}-$ CoO@NC/NC-800) could exhibit an ORR onset potential of $0.961 \mathrm{~V} v s$. RHE and a half-wave potential of $0.868 \mathrm{~V} v s$. RHE. These values are higher than the onset and half-wave potentials of the single component $\mathrm{Co}$ and $\mathrm{CoO}$ based catalysts and even close to those of the commercial $\mathrm{Pt} / \mathrm{C}$, strongly suggesting that the $\mathrm{Co}-\mathrm{CoO} @ \mathrm{NC} / \mathrm{NC}$ is an efficient catalyst for the ORR. Up to now, although a significant amount of the catalysts has been reported to be catalytically active for the ORR, those with activities higher than or even close to the commercial Pt/C has less been reported. The work presented here is therefore of great interest since it develops a new and high efficient catalyst using a simple synthetic method and would be also helpful to design other catalysts.

\section{Experimental}

\section{Chemicals and reagents}

Urea (99\%) and glucose (99\%) were obtained from Guangzhou Chemical Reagent Co. Ltd. Cobalt(II) nitrate hexahydrate $\left(\mathrm{Co}\left(\mathrm{NO}_{3}\right)_{2} \cdot 6 \mathrm{H}_{2} \mathrm{O}, 99 \%\right)$ was obtained from Tianjin Kermel Chemical Reagent Co. Ltd. Potassium hydroxide (KOH, 85\%), methanol and isopropanol were obtained from Tianjin chemical reagent Co. Ltd. Nafion (5.0 wt\%) was purchased from DuPont Company. All the chemicals were used as received without further purification. Deionized (DI) water with a resistance of $\sim 18 \mathrm{M} \Omega \mathrm{cm}^{-1}$ was used in all the reactions.

\section{Material synthesis}

In a typical synthesis, $50.0 \mathrm{mg}$ of glucose and $1.0 \mathrm{~g}$ of urea were dissolved with $40 \mathrm{~mL}$ of DI water. The obtain mixture was stirred vigorously for $30 \mathrm{~min}$, followed by addition of $0.6 \mathrm{mmol}$ of $\mathrm{Co}\left(\mathrm{NO}_{3}\right)_{2}$. The obtained pink homogenous solution was heated at $80{ }^{\circ} \mathrm{C}$ under stirring to remove water. After vaporization, the obtained solid was loaded on a crucible, then thoroughly dried at $200{ }^{\circ} \mathrm{C}$ for $2 \mathrm{~h}$, and finally calcined at 700,800 or $900^{\circ} \mathrm{C}$ for $3 \mathrm{~h}$ in a tube furnace under the $\mathrm{N}_{2}$ flow. The products obtained from the calcination temperature of 700,800 and $900{ }^{\circ} \mathrm{C}$ were named as Co-CoO@NC/NC-700, Co-CoO@NC/NC-800, and Co-CoO@NC/ NC-900, respectively. The same synthetic procedure was performed for the synthesis of the $\mathrm{Co}-\mathrm{CoO} @ \mathrm{C} / \mathrm{C}-800$ and the $\mathrm{NC}$ in the absence of urea or $\mathrm{Co}\left(\mathrm{NO}_{3}\right)_{2}$, respectively, while keeping other parameters constant.

\section{Physical characterizations}

X-ray diffraction patterns (XRD) of the samples were recorded on a Bruker D8 X-ray diffractometer with the $\mathrm{Cu} \mathrm{K} \alpha$ radiation and were analyzed using Bruker EVA and Bruker TopAs 4.2 software. Scanning electron microscopy (SEM) was performed on Merlin with an operation voltage of $20.0 \mathrm{kV}$. Transmission electron microscopy (TEM) and high-resolution transmission electron microscopy (HRTEM) measurements were performed on a JEM$2100 \mathrm{~F}$ with an accelerating voltage of $200 \mathrm{kV}$. Raman spectra were collected on a LabRAM HR Evolution system using an Ar laser with excitation wavelength of $514.5 \mathrm{~nm}$. The chemical compositions of the samples were determined by X-ray photoelectron spectroscopy (XPS) performed on a PHI X-tool (Japan) with an $\mathrm{Al} \mathrm{K} \alpha$ X-ray radiation (1486 eV). The Brunauer-EmmettTeller (BET) surface areas and pore size distributions of the samples were determined by the $\mathrm{N}_{2}$ adsorption/desorption isotherms recorded at $77 \mathrm{~K}$ using Micromeritics ASAP 2020. 


\section{Electrochemical measurement}

A standard three-electrode cell was used for the electrochemical measurements using an electrochemical workstation (CHI 760D, China). Platinum wire and a saturated calomel electrode served as the counter and the reference electrodes, respectively. The working electrode was prepared by dropping drops of the catalyst ink onto the glassy carbon electrode and then naturally dried at room temperature. The catalyst ink with a catalysts concentration of $2 \mathrm{mg} \mathrm{mL} \mathrm{m}^{-1}$ was prepared by dispersing the catalyst and $10 \mu \mathrm{L}$ of Nafion ( $5 \mathrm{wt} \%$ ) into a mixture of water and isopropanol with a volume ratio of $1.0: 3.0$, followed by ultrasonication to obtain a homogeneous solution. The loading of the catalyst on the working electrode was well controlled to $\sim 0.1 \mathrm{mg} \mathrm{cm}^{-2}$. The working electrode with the $\mathrm{Pt} / \mathrm{C}(20 \mathrm{wt} \% \mathrm{Pt}$ on the carbon, Johnson Matthey) used for comparison was prepared in a similar manner. The cyclic voltammograms (CVs) were recorded at ambient temperature in an $0.1 \mathrm{M} \mathrm{KOH}$ solution. Before the measurements, the solution was aerated with $\mathrm{N}_{2}$ or $\mathrm{O}_{2}$ for at least $30 \mathrm{~min}$. The $\mathrm{N}_{2}$ or $\mathrm{O}_{2}$ flow was maintained above the solution to keep the $\mathrm{N}_{2}$ or $\mathrm{O}_{2}$ saturation during the electrochemical measurements. The linear sweep voltammograms (LSVs) obtained from the rotating disk electrode (RDE) at the different rotating rates were collected in the $\mathrm{O}_{2}$ saturated $0.1 \mathrm{M} \mathrm{KOH}$ at a rate of $5 \mathrm{mV} \mathrm{s}^{-1}$ from $0.3 \mathrm{~V}$ to $-0.8 \mathrm{~V}$ (vs. SCE). The kinetic analysis was performed using the Koutecky-Levich (K-L) equations shown below:

$$
\begin{aligned}
& \frac{1}{j}=\frac{1}{j_{\mathrm{k}}}+\frac{1}{j_{\mathrm{L}}}=\frac{1}{j_{\mathrm{k}}}+\frac{1}{B \omega^{1 / 2}} \\
& B=0.62 n F C_{\mathrm{O}} D^{2 / 3} v^{-1 / 6}
\end{aligned}
$$

where $j, j_{\mathrm{k}}$, and $j_{\mathrm{L}}$ represent the measured current, the kinetic current, and the limiting current densities, respectively. $C_{\mathrm{O}}$ is the concentration of molecular oxygen in $\mathrm{O}_{2}$-saturated $0.1 \mathrm{M} \mathrm{KOH}$ $\left(C_{\mathrm{O}}=1.2 \times 10^{-6} \mathrm{~mol} \mathrm{~cm}{ }^{-3}\right)$, and $\omega$ the electrode rotating rate, $B$ the Levich constant, $F$ the Faraday constant $\left(F=96500 \mathrm{C} \mathrm{mol}^{-1}\right), n$ transferred electron number, $D$ the $\mathrm{O}_{2}$ diffusion coefficient of the electrolyte $\left(D=1.9 \times 10^{-5} \mathrm{~cm}^{-2} \mathrm{~s}^{-1}\right)$, $v$ the kinetic viscosity $\left(v=0.01 \mathrm{~cm}^{2} \mathrm{~s}^{-1}\right)$.

The rotating ring-disk electrode (RRDE) technique was also employed to analyze the ORR properties of the catalysts. The electron transfer number and the amount of $\mathrm{HO}_{2}{ }^{-}$generated during ORR from RRDE test were determined through the equations below:

$$
\begin{gathered}
\mathrm{HO}_{2}(\%)=\frac{200 I_{\mathrm{R}}}{\left|I_{\mathrm{D}}\right| N+I_{\mathrm{R}}} \\
n=\frac{4\left|I_{\mathrm{D}}\right|}{\left|I_{\mathrm{D}}\right|+I_{\mathrm{R}} / N}
\end{gathered}
$$

where $I_{\mathrm{R}}$ is the ring current, $I_{\mathrm{D}}$ is the disk current, $N$ is the collection efficiency with a value of 0.4 . The potential reported in this work was all referenced to RHE. In the $0.1 \mathrm{M} \mathrm{KOH}$ solution, the potentials versus the SCE reference electrode were converted to the RHE through the calibration equation: $E_{\mathrm{RHE}}=$ $E_{\mathrm{SCE}}+0.2438+0.0591 \times 13$.

\section{Results and discussion}

Fig. 1a shows a typical SEM image of the Co-CoO@NC/NC-800 synthesized by a procedure involving the drying of the reaction mixture of $\mathrm{Co}\left(\mathrm{NO}_{3}\right)_{2}$, glucose and urea at $200{ }^{\circ} \mathrm{C}$ and its subsequent calcination at the high temperature of $800{ }^{\circ} \mathrm{C}$. It reveals that the $\mathrm{Co}-\mathrm{CoO} @ \mathrm{NC} / \mathrm{NC}-800$ has a morphology resembling to the crumpled papers with the deposition of the small sized particles. This is also verifiable by its TEM image in Fig. 1b, which shows a structure consisting of the sheet-like carbon material and uniformly sized nanoparticles. The average size of the nanoparticles is $\sim 14.8 \mathrm{~nm}$, as estimated by the particle size distribution histogram in Fig. 1c. The highresolution TEM image of the Co-CoO@NC/NC-800 in Fig. 1d indicates that the nanoparticles are composed of mixed phases of $\mathrm{Co}$ and $\mathrm{CoO}$. As shown in Fig. 1d, the lattice fringes corresponding to the (111) and (200) planes of the face-centered cubic (fcc) structure cobalt and the (220) plane of the rock salt cubic structure $\mathrm{CoO}$ could be identified. The presence of the mixed phases of $\mathrm{Co}$ and $\mathrm{CoO}$ suggests that a fraction of $\mathrm{Co}^{2+}$ has been reduced by glucose and urea or their calcination products during the calcination process, while the residual has changed into $\mathrm{CoO}$ through the decomposition of $\mathrm{Co}\left(\mathrm{NO}_{3}\right)_{2}$ or through its reaction with oxygen from the decomposed glucose and urea. Most interestingly, each $\mathrm{Co} / \mathrm{CoO}$ nanoparticle is surrounded by a crystallized shell of $1.2 \mathrm{~nm}$ (Fig. 1d). The $d$-spacing measured from the lattice fringes of the shell is 0.335 , well consistent with the interlayer spacing of the (002) plane of the graphitic carbon. This suggests that the $\mathrm{Co} / \mathrm{CoO}$ nanoparticles are coated with the carbon shells. These carbon shells could be attributed to the carbonized product of glucose and urea formed during the high-temperature calcination processes.

The X-ray diffraction (XRD) pattern of the Co-CoO@NC/NC800 is given in Fig. 2a. It shows three distinct peaks at $2 \theta=$ $44.2^{\circ}, 51.6^{\circ}$ and $75.8^{\circ}$, corresponding to the (111), (200), and (220) reflections of the fcc structure metallic Co (PDF no. 150806), and two weak peaks $2 \theta=36.6$ and $42.3^{\circ}$, corresponding to the (111) and (200) reflections of the rock salt cubic structure CoO (PDF no. 48-1719). This further demonstrates the coexistence of $\mathrm{Co}$ and $\mathrm{CoO}$ in the Co-CoO@NC/NC-800. The reason that the $\mathrm{Co}-\mathrm{CoO} @ \mathrm{NC} / \mathrm{NC}-800$ exhibits low diffraction peaks corresponding to $\mathrm{CoO}$ could be attributed to its relatively low content in comparison to that of the metallic cobalt (as demonstrated by the XPS results shown below) and the low Xray scattering factor of cobalt oxide. Besides these peaks, the XRD pattern of the Co-CoO@NC/NC-800 also exhibits a broad peak at $2 \theta=25.9^{\circ}$, which could be assigned to the (002) reflection of the layer-by-layer aligned graphitic nanosheets, suggesting the presence of the graphitic carbon in the CoCoO@NC/NC-800. This could be further corroborated by the Raman spectrum of the Co-CoO@NC/NC-800 in Fig. 2b, which shows pronounced peaks at $1350 \mathrm{~cm}^{-1}$ and $1580 \mathrm{~cm}^{-1}$, assignable to the $\mathrm{D}$ band (arising from the disorder carbon) and $\mathrm{G}$ band (associated with tangential stretching mode of highly order graphitic carbon) of the graphitic structure. Worthnoting is that the Raman spectrum of the Co-CoO@NC/NC-800 also 

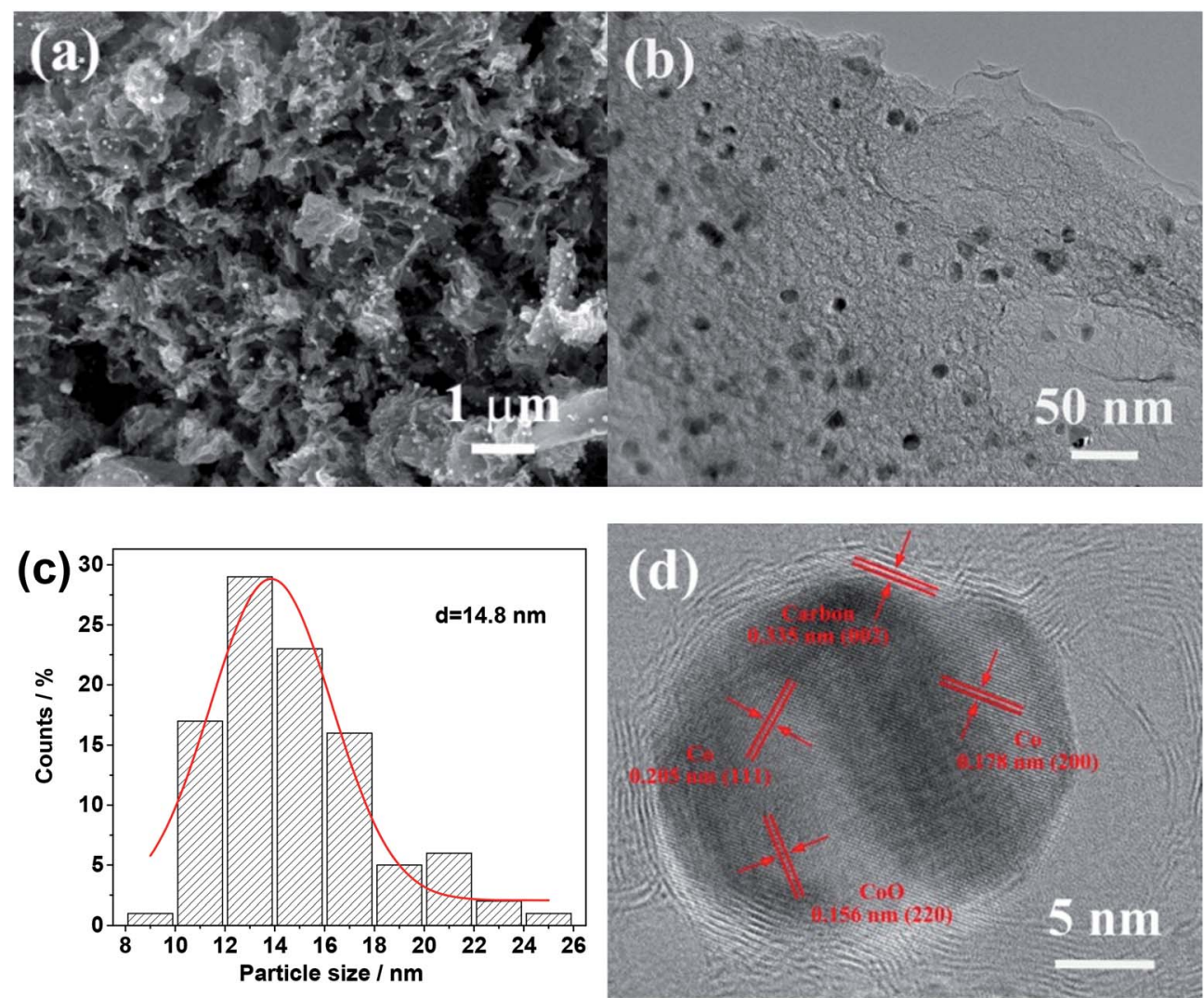

Fig. 1 . (a) SEM and (b) TEM images of the Co-CoO@NC/NC-800. (c) Particle size distribution histogram of the Co-CoO NPs in the CoCoO@NC/NC-800. (d) High resolution TEM image of the Co-CoO@NC/NC-800.

exhibits peaks at 473,517 , and $679 \mathrm{~cm}^{-1}$, assignable to active vibrational modes $\mathrm{E}_{g}, \mathrm{~F}_{2 \mathrm{~g}}$, and $\mathrm{A}_{1 \mathrm{~g}}$ of the cubic structure $\mathrm{CoO}$, respectively ${ }^{38,39}$ This further supports the presence of $\mathrm{CoO}$ in the $\mathrm{Co}-\mathrm{CoO} @ \mathrm{NC} / \mathrm{NC}-800$.

The XPS measurements were carried out to gain information on the elemental composition of the Co-CoO@NC/NC-800. Fig. 3a shows the XPS survey spectrum of the Co-CoO@NC/ $\mathrm{NC}-800$, which indicates that the $\mathrm{Co}-\mathrm{CoO} @ \mathrm{NC} / \mathrm{NC}-800$ is composed of $\mathrm{Co}, \mathrm{O}, \mathrm{N}$, and $\mathrm{C}$. The deconvolution of the Co $2 \mathrm{p}$ spectrum in Fig. $3 \mathrm{~b}$ clearly demonstrates the presence of both $\mathrm{Co}^{(0)}$ and $\mathrm{Co}^{(\mathrm{II})}$ in the Co-CoO@NC/NC-800. As shown in Fig. 3b, the peaks corresponding to $\mathrm{Co} 2 \mathrm{p}_{3 / 2}$ and $2 \mathrm{p}_{1 / 2}$ of $\mathrm{Co}^{(\mathrm{II})}$ could be observed at $780.5 \mathrm{eV}$ and $796.5 \mathrm{eV}$, respectively, while the peak corresponding to Co $2 \mathrm{p}_{3 / 2}$ and $2 \mathrm{p}_{1 / 2}$ of $\mathrm{Co}^{(0)}$ is observable at $778.8 \mathrm{eV}$ and $794.1 \mathrm{eV}$, respectively. Based on the deconvoluted spectrum shown in Fig. $3 \mathrm{~b}$, the relative atomic ratio of $\mathrm{Co}^{(0)}: \mathrm{Co}^{(\mathrm{II})}$ is estimated to $\sim 3: 2$. It implies that Co in the CoCoO@NC/NC-800 mainly exists in the metallic form. Fig. 3c displays the deconvoluted $\mathrm{C}$ 1s spectrum of the $\mathrm{Co}-\mathrm{CoO} @ \mathrm{NC} /$ NC-800, which shows four components at binding energies of $284.5,285.3,286.3$, and $288.2 \mathrm{eV}$, corresponding to $\mathrm{sp}^{2} \mathrm{C}, \mathrm{C}=\mathrm{N} /$ $\mathrm{C}-\mathrm{O}, \mathrm{C}-\mathrm{O}-\mathrm{C} / \mathrm{C}-\mathrm{N}$, and $\mathrm{O}-\mathrm{C}=\mathrm{O}$, respectively. The domination of the peak corresponding to $\mathrm{sp}^{2} \mathrm{C}$ suggests the carbon in the CoCoO@NC/NC-800 is well graphitized. This is in line with the result shown by the Raman spectrum (Fig. 2b), which exhibits pronounced peaks corresponding to the $\mathrm{D}$ and $\mathrm{G}$ bands of the
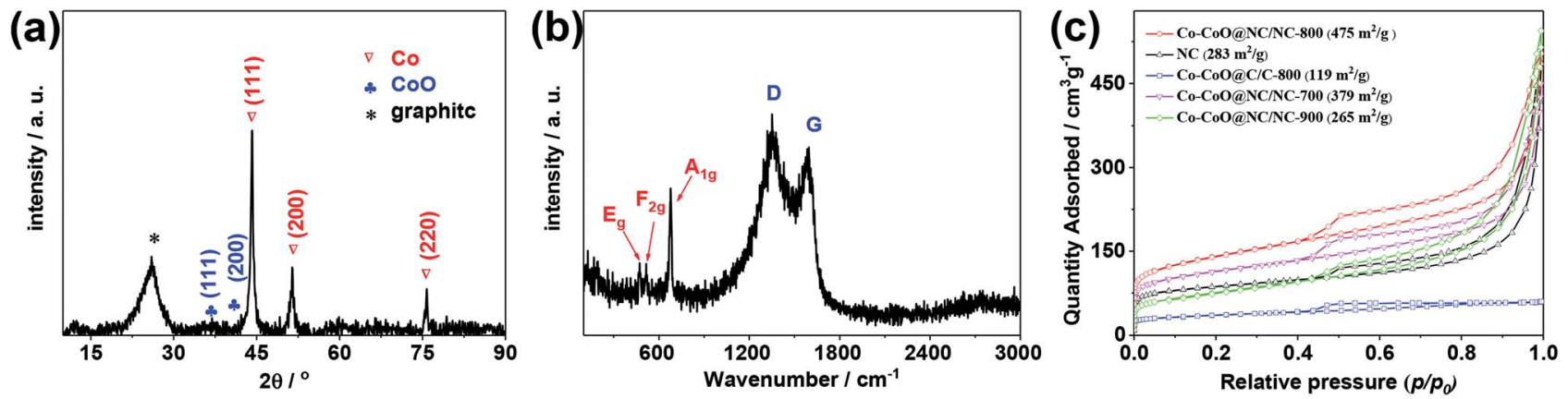

Fig. 2 (a) XRD patterns, (b) Raman spectra of the Co-CoOaNC/NC-800. (c) $\mathrm{N}_{2}$ adsorption/desorption isotherms of the Co-CoOaNC/NC-800, the NC, the Co-CoOaC/C-800, the Co-CoOaNC/NC-700, the Co-CoOaNC/NC-900. 
graphitic structure. The existence of the peaks corresponding to $\mathrm{C}=\mathrm{N}$ and $\mathrm{C}-\mathrm{N}$ in the deconvoluted $\mathrm{C} 1$ s spectrum indicates that carbon in the Co-CoO@NC/NC-800 is doped with nitrogen. This could be also verified by the deconvoluted $\mathrm{N}$ 1s spectrum in Fig. 3d, which shows four peaks at binding energies of 397.9, 399.6, 400.7, and 402.3 eV, assignable to pyridinic, pyrrolic, graphitic, and oxidized nitrogen, respectively. This is similar to the NC reported previously. ${ }^{\mathbf{4 0 , 4 1}}$ Since the Co-CoO@NC/NC-800 was synthesized from a mixture, in which the reactants were well dispersed, we believe that both the carbon shell of the Co$\mathrm{CoO}$ nanoparticles and the carbon sheet observed in the SEM and TEM images of the Co-CoO@NC/NC-800 (Fig. 1a and b) are doped with nitrogen. The elemental composition of the CoCoO@NC/NC-800 could be further demonstrated by its elemental mapping image in Fig. $\mathrm{S} 1, \dagger$ which clearly shows the uniform distribution of $\mathrm{Co}, \mathrm{O}, \mathrm{N}$, and $\mathrm{C}$. Based on the XPS results and the TGA curve of the Co-CoO@NC/NC-800 in Fig. $\mathrm{S} 2, \dagger$ the weight percentage of $\mathrm{Co}-\mathrm{CoO}$ in the $\mathrm{Co}-\mathrm{CoO} @ \mathrm{NC} /$ NC-800 is estimated to be $28.7 \%$ (the detailed calculation is given in the ESI $\dagger$ ).

The results shown above make it clear that the Co-CoO@NC/ NC-800 has a structure consisting of the sheet-like NC with the deposition of the NC coated $\mathrm{Co}-\mathrm{CoO}$ nanoparticles. To understand the role of urea in the formation of the Co-CoO@NC/NC800 , the same synthetic procedure was performed in the absence of urea while keeping other parameters constant. It shows the formation of the Co-CoO@C/C-800 consisting of largely sized $\mathrm{Co}-\mathrm{CoO}$ particles with a big inhomogeneity in the size distribution (the TEM image, XPS spectra, XRD diffraction pattern, and Raman spectrum of the Co-CoO@C/C-800 is given in Fig. S3a, S4, S5, and S6, † respectively). This suggests that urea in the reaction mixture does not simply serve as the nitrogen source for the doping of carbon, but plays an important role in promoting the formation the $\mathrm{Co}-\mathrm{CoO} @ \mathrm{NC} / \mathrm{NC}-800$ with uniformly nanosized $\mathrm{Co}-\mathrm{CoO}$ particles. It is imaginable that during the high temperature calcinations the explosive pyrolysis of urea would facilitate the doping of nitrogen into graphitic structure of carbon with different forms, such as pyridinic, pyrrolic, graphitic, and oxidized nitrogen. Due to the high electronegativity of nitrogen, the doping sites in the forms of pyridinic, pyrrolic, and graphitic nitrogen are negatively charged, ${ }^{42,43}$ making them capable of adsorbing the $\mathrm{Co}^{2+}$ ions through the coordination and electrostatic interactions. This would prevent the aggregation of the $\mathrm{Co}^{2+}$ ions during its transformation into $\mathrm{Co}-\mathrm{CoO}$ nanoparticles, facilitating the formation of the Co-CoO nanoparticles with a uniform and small size.

The calcination temperature in the synthetic procedure is found to have a profound effect on the final product. As demonstrated by the TEM image of the Co-CoO@NC/NC-700 in Fig. S3b, $\uparrow$ the low temperature calcination would lead to the formation of smaller $\mathrm{Co}-\mathrm{CoO}$ nanoparticles with a more uniform size. The XPS spectrum in Fig. S4 and Table S1† show that the Co-CoO@NC/NC-700 contains relatively higher atomic percentages of oxygen and nitrogen, suggesting that the urea and glucose are not well carbonized at the low calcination
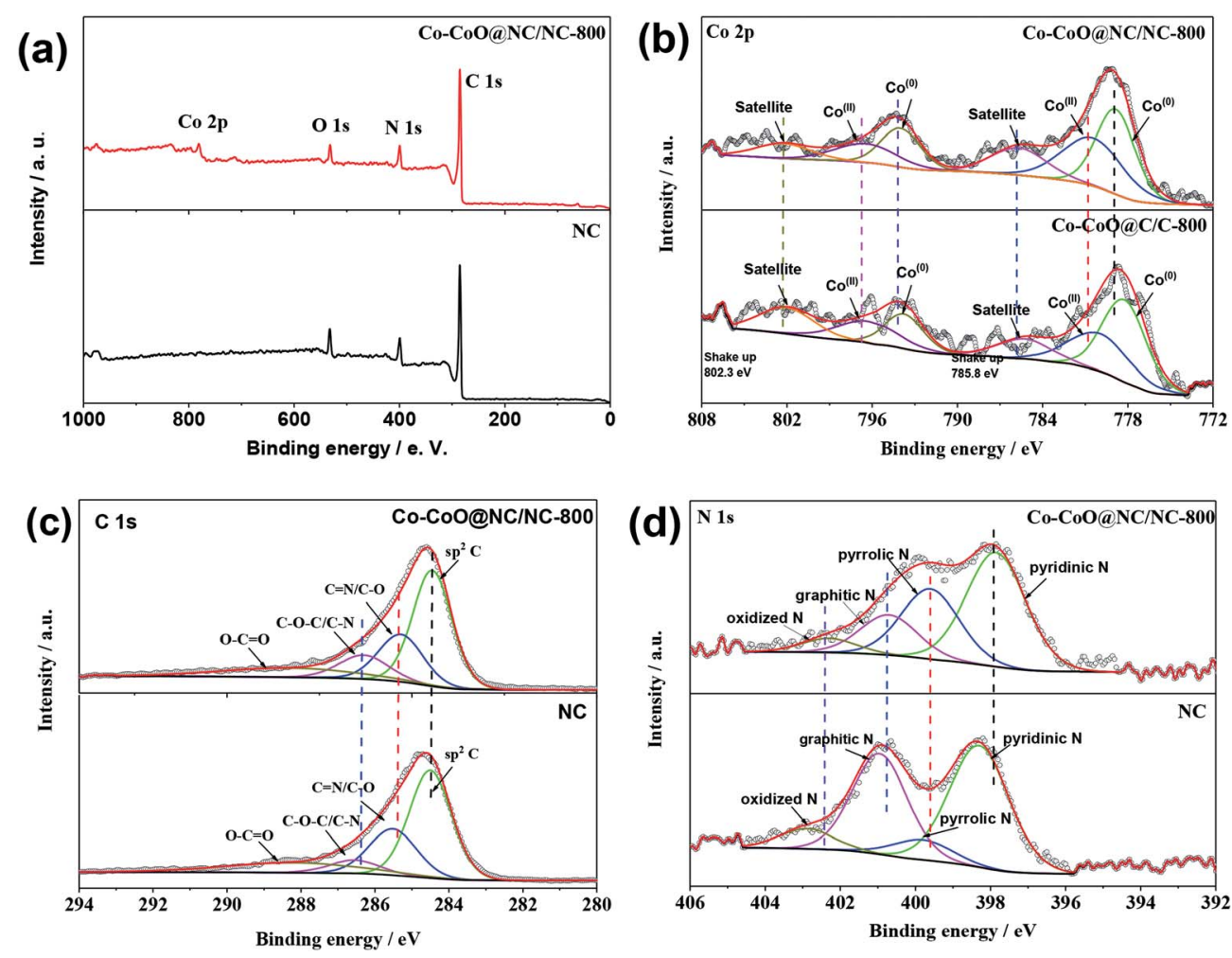

Fig. 3 (a) XPS survey spectra of the Co-CoOaNC/NC-800 and the NC. (b) High resolution Co 2p spectra of the Co-CoO@NC/NC-800 and the Co-CoOaC/C-800. (c) High resolution C 1s spectra of the Co-CoO@NC/NC-800 and the NC. (d) High resolution N 1s spectra of the CoCoO@NC/NC-800 and the NC. 
temperature. On the other hand, when the reaction mixture was calcined at the high temperature of $900{ }^{\circ} \mathrm{C}$, the formation of the $\mathrm{Co}-\mathrm{CoO}$ nanoparticles with the relatively larger size and big size inhomogeneity is observed, as indicated by the TEM image of the Co-CoO@NC/NC-900 in Fig. S3c. $\uparrow$ This indicates that the mild reaction condition at the lower temperature is favorable for the formation of the Co-CoO nanoparticles with small and uniform sizes, while at the higher calcination temperature, the improved uncontrollability of the decomposition of $\mathrm{Co}\left(\mathrm{NO}_{3}\right)_{2}$, glucose, and urea due to the increase of the pyrolysis rates would promote the formation of large $\mathrm{Co}-\mathrm{CoO}$ nanoparticles with a big inhomogeneity in the size distribution (the XPS spectrum, XRD diffraction pattern, and Raman spectra of the Co-CoO@NC/NC-900 is given in Fig. S4, S5, and S6, $\dagger$ respectively). Table S1† shows that the Co-CoO@NC/NC-900 contains relatively lower atomic percentages of oxygen and nitrogen, suggesting that the high calcination temperature would decrease the contents of oxygen and nitrogen containing groups in the final product. This is well consistent with the results reported previously, ${ }^{\mathbf{4 4}, 45}$ which showed that the high calcination temperature would lead to the significant decomposition of nitrogen and oxygen containing groups in the NC. Additionally, we also found that the drying step in the synthetic procedure is necessary to obtain the Co-CoO@NC/NC-800 with uniformly sized Co-CoO nanoparticles. As shown in Fig. S3d, $\dagger$ when the reaction mixture was directly calcined at $800{ }^{\circ} \mathrm{C}$, the significant aggregation of the $\mathrm{Co}-\mathrm{CoO}$ nanoparticles is observed in the final product. This might be attributed to the highly corrosive nature of water vapor at the high temperature. Its presence in the reaction mixture would greatly accelerate the pyrolysis of $\mathrm{Co}\left(\mathrm{NO}_{3}\right)_{3}$, glucose, and urea, leading to uncontrollable aggregation and coalescence of the $\mathrm{Co}-\mathrm{CoO}$ nanoparticles during their nucleation and growth.

The textural property of the Co-CoO@NC/NC-800 was investigated by the $\mathrm{N}_{2}$ adsorption/desorption isotherms. As shown in Fig. 2c, the $\mathrm{N}_{2}$ adsorption/desorption isotherms of the Co-CoO@NC/NC-800 exhibit a rapid uptake at $p / p_{0}<0.04$ followed by a H3-type hysteresis in the mesopore region. This indicates the co-existence of both the micropores and macropores in the sample. Additionally, the big uptake of $\mathrm{N}_{2}$ at $p / p_{0}>0.9$ in the adsorption/desorption isotherms also demonstrates the existence of macropores in the Co-CoO@NC/NC800. Base on the $\mathrm{N}_{2}$ adsorption/desorption isotherms in Fig. 2c, the specific surface area of the Co-CoO@NC/NC-800 estimated using the BET method is $475 \mathrm{~m}^{2} \mathrm{~g}^{-1}$. The presence of urea in the reaction precursor is found to have a significant effect on the BET specific surface area of the sample. As shown in Fig. 2c, the Co-CoO@C/C exhibits significantly lower BET specific surface area than that of the Co-CoO@NC/NC-800. This might be due to the reason that the thermal decomposition of urea during the calcination would produce a large amount of gases, such as $\mathrm{CO}_{2}, \mathrm{NH}_{3}$, and other gaseous products, ${ }^{46,47}$ which are helpful to the formation of the porous structure in the $\mathrm{Co}-$ CoO@NC/NC-800. Most interestingly, although the CoCoO@NC/NC-700 and the Co-CoO@NC/NC-900 exhibit the $\mathrm{N}_{2}$ adsorption/desorption isotherms comparable to those of the Co-CoO@NC/NC-800, their BET specific surface areas are much lower than that of the Co-CoO@NC/NC-800. This suggests that the calcination temperature also has a great effect on the BET specific surface area of the sample. As demonstrated above, the calcination temperature influences the average size of $\mathrm{Co}-\mathrm{CoO}$ nanoparticles and the doping content of nitrogen in the $\mathrm{NC}$, and it also affects the pyrolysis rate of $\mathrm{Co}\left(\mathrm{NO}_{3}\right)_{2}$, glucose, and urea. These might be the reasons leading to the variations of the BET specific surface areas of the samples with the calcination temperature. The high specific BET surface area is helpful for the application of the Co-CoO@NC/NC-800 as the electrocatalyst for the ORR, since it allows for its good accessibility to the electrolyte.

The catalytic activity of the Co-CoO@NC/NC-800 for the ORR is first assessed by its CV curves in the $\mathrm{N}_{2}$ or $\mathrm{O}_{2}$ saturated $0.1 \mathrm{M}$ $\mathrm{KOH}$ solution. As shown in Fig. 4a, the CV curve of the CoCoO@NC/NC-800 in the $\mathrm{N}_{2}$ saturated $0.1 \mathrm{M} \mathrm{KOH}$ solution shows featureless voltammetric currents, while that in the $\mathrm{O}_{2}$ saturated 0.1 M KOH solution shows a well-defined cathodic peak corresponding to the reduction of oxygen. This clearly demonstrates the catalytic activity of the Co-CoO@NC/NC-800 toward the ORR. Fig. 4b displays the LSV of the Co-CoO@NC/ $\mathrm{NC}-800$ for the ORR in the $\mathrm{O}_{2}$ saturated $0.1 \mathrm{M} \mathrm{KOH}$ solution. It clearly shows that the Co-CoO@NC/NC-800 can exhibit an ORR onset potential of $0.961 \mathrm{~V} v s$. RHE and half-wave potential of $0.868 \mathrm{~V} v s$. RHE. Most interestingly, the ORR onset and halfwave potentials of the Co-CoO@NC/NC-800 are very close to those of the $\mathrm{Pt} / \mathrm{C}$ (the ORR onset and half-wave potential of the $\mathrm{Pt} / \mathrm{C}$ are 0.962 and $0.861 \mathrm{~V} v$ s. RHE, respectively, which are close to the reported values ${ }^{\mathbf{4 8 , 4 9}}$ ). This clearly suggests that the Co$\mathrm{CoO}$ @NC/NC-800 is an efficient electrocatalyst for the ORR. Comparison in Fig. 4d shows that the Co-CoO@NC/NC-800 can even exhibit the ORR half-wave potential higher than those of most catalysts reported previously (Table $\mathrm{S} 2 \dagger$ shows a more detailed comparison of the ORR onset and half-wave potentials), strongly suggesting that the Co-CoO@NC/NC-800 is one of the best ORR catalysts among those reported (Table 1).

To understand the origin of the high ORR activity of the CoCoO@NC/NC-800, the LSVs of the NC (synthesized from a same synthetic procedure while in the absence of $\left.\mathrm{Co}\left(\mathrm{NO}_{3}\right)_{2}\right)$, the Co$\mathrm{CoO} @ \mathrm{C} / \mathrm{C}-800$, the $\mathrm{Co}-\mathrm{CoO} @ N C / \mathrm{NC}-700$, and the $\mathrm{Co}-$ CoO@NC/NC-900 in the $\mathrm{O}_{2}$ saturated 0.1 M KOH solutions were also recorded. Fig. $4 \mathrm{a}$ and $\mathrm{b}$ shows that although the NC is electrochemically active for the ORR, its activity is much lower than that of the Co-CoO@NC/NC-800. This clearly indicates a great importance of the Co-CoO nanoparticles in the high catalytic activity of the Co-CoO@NC/NC-800. The nitrogen doped structure of the carbon is also found to be important in improving its catalytic activity of the Co-CoO@NC/NC-800 for the ORR. As shown in Fig. 4a and b, the Co-CoO@C/C-800 exhibits significantly lower catalytic activity for the ORR than that of the Co-CoO@NC/NC-800. Previous work has demonstrated that the NC could be also highly active for the ORR, when its composition is well controlled. ${ }^{50-53}$ We therefore assume that the high catalytic activity of the Co-CoO@NC/NC800 is a result of a synergistic effect between two electroactive catalysts of the Co-CoO nanoparticles and the NC. Worthnoting is that the catalytic activity of the sample is also effected by the 

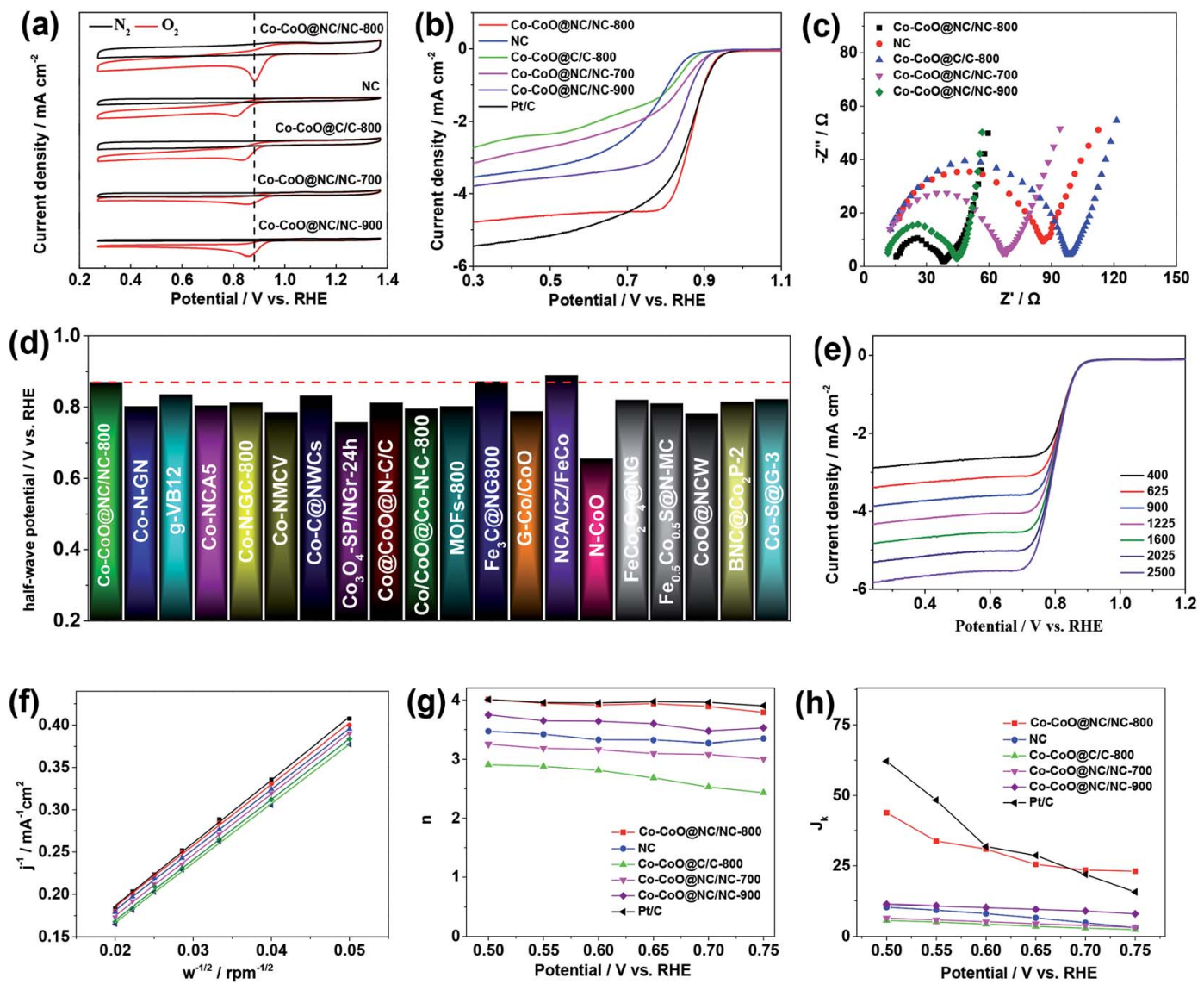

Fig. 4 (a) CV curves of the Co-CoO@NC/NC-800, the NC, the Co-CoO@C/C-800, the Co-CoO@NC/NC-700, and the Co-CoO@NC/NC900 in $\mathrm{O}_{2}$ or $\mathrm{N}_{2}$ saturated $0.1 \mathrm{M} \mathrm{KOH}$ solution at a scan rate of $5 \mathrm{mV} \mathrm{s}^{-1}$ (b) LSVs for the ORR by the Co-CoO@NC/NC-800, the NC, the Co$\mathrm{CoO}$ aC/C-800, the Co-CoO@NC/NC-700, the Co-CoOaNC/NC-900, and the $\mathrm{Pt} / \mathrm{C}$ in an $\mathrm{O}_{2}$-saturated $0.1 \mathrm{M} \mathrm{KOH}$ solution at a scan rate of $5 \mathrm{mV} \mathrm{s}^{-1}$ (c) EIS spectra of the Co-CoO@NC/NC-800, the NC, the Co-CoOaC/C-800, the Co-CoO@NC/NC-700, and the Co-CoOaNC/NC900. (d) Comparison of the ORR half-wave potential of the Co-CoOaNC/NC-800 with those of the catalysts reported. (e) LSVs for the ORR by the $\mathrm{Co}-\mathrm{CoO}$ aNC/NC-800 in an $\mathrm{O}_{2}$-saturated $0.1 \mathrm{M} \mathrm{KOH}$ solution at various rotation rates at a scan rate of $5 \mathrm{mV} \mathrm{s}^{-1}$ (f) $\mathrm{K}-\mathrm{L}$ plots of the ORR by the $\mathrm{Co}-\mathrm{CoO} a \mathrm{NC} / \mathrm{NC}-800$. (g) Electron transfer number and (h) kinetic current density of the ORR by the Co-CoO@NC/NC-800, the NC, the Co-CoOaC/C-800, the Co-CoOaNC/NC-700, the Co-CoOaNC/NC-900 and the Pt/C.

calcination temperature. As shown in Fig. $4 \mathrm{a}$ and b, the catalytic activity of the $\mathrm{Co}-\mathrm{CoO} @ \mathrm{NC} / \mathrm{NC}-800$ is higher than those of the Co-CoO@NC/NC-700 and Co-CoO@NC/NC-900. This might be due to the fact that the $\mathrm{Co}-\mathrm{CoO} @ \mathrm{NC} / \mathrm{NC}-800$ synthesized at the calcination temperature of $800{ }^{\circ} \mathrm{C}$ has small and uniformly sized $\mathrm{Co}-\mathrm{CoO}$ nanoparticles and exhibits a higher BET specific surface area, which expose more active sites and increase the accessibility of the Co-CoO@NC/NC-800 to the ORR. Additionally, the $\mathrm{Co}-\mathrm{CoO} @ \mathrm{NC} / \mathrm{NC}-800$ has a well carbonized structure with the doping of an appropriate amount of nitrogen, which increases the electrical conductivity and hydrophilicity of the Co-CoO@NC/NC-800. Fig. 4c shows the electrochemical impedance spectra (EISs) of the Co-CoO@NC/NC-800, the NC, the $\mathrm{Co}-\mathrm{CoO} @ \mathrm{C} / \mathrm{C}-800$, the $\mathrm{Co}-\mathrm{CoO} @ \mathrm{NC} / \mathrm{NC}-700$, and the $\mathrm{Co}-$ $\mathrm{CoO} @ \mathrm{NC} / \mathrm{NC}-900$, which clearly demonstrate that the $\mathrm{Co}^{-}$ CoO@NC/NC-800 has higher electrical conductivity.

The work reported previously has suggest that the deposition of transition metal based catalysts onto the surface of carbon would impose a strong electronic coupling between two materials, which could also improve the catalytic activities of the composite materials. ${ }^{48}$ The nitrogen doping could promote the electronic coupling between transition metal based catalysts and carbon..$^{39,48}$ It is therefore expectable that the strong electronic coupling between the $\mathrm{Co}-\mathrm{CoO}$ nanoparticles and the NC is an additional reason leading to the high catalytic activity of the Co-CoO@NC/NC-800 for the ORR. To demonstrate the presence of the electronic coupling between the $\mathrm{Co}-\mathrm{CoO}$ nanoparticles and the NC, the XPS spectra of the NC were carefully analyzed. The XPS survey spectra in Fig. 3a shows the absence of the peak corresponding to Co in the NC. Most notably, although the high-resolution $\mathrm{N} 1 \mathrm{~s}$ spectrum of the NC exhibits the components similar to that of the Co-CoO@NC/ NC-800, their peak positions are slightly shifted to the lower binding energies, as shown in Fig. 3d. This clearly corroborates the presence of the strong electronic coupling between the Co$\mathrm{CoO}$ nanoparticles and the $\mathrm{NC}$ with a possible electron transfer from the $\mathrm{Co}-\mathrm{CoO}$ nanoparticles to the NC. Indeed, the strong electronic coupling between the $\mathrm{Co}-\mathrm{CoO}$ nanoparticles and the NC could also be demonstrated by the $\mathrm{C} 1 \mathrm{~s}$ spectra in Fig. 3c, which shows the shifts of the peak positions of $\mathrm{C}=\mathrm{N} / \mathrm{C}-\mathrm{O}$, and 
Table 1 Comparison of the ORR catalytic activities of the CoCoO@NC/NC-800, the NC, the Co-CoOaC/C-800, the CoCoO@NC/NC-700, the Co-CoO@NC/NC-900, and the Pt/C

\begin{tabular}{lll}
\hline Catalyst & $\begin{array}{l}\text { Onset potential/V } \\
\text { vs. } \mathrm{RHE}^{a}\end{array}$ & $\begin{array}{l}\text { Half-wave potential/V } \\
\text { vs. RHE }\end{array}$ \\
\hline Co-CoO@NC/NC-800 & 0.961 & 0.868 \\
NC & 0.889 & 0.777 \\
Co-CoO@C/C-800 & 0.899 & 0.817 \\
Co-CoO@NC/NC-700 & 0.921 & 0.841 \\
Co-CoO@NC/NC-900 & 0.920 & 0.842 \\
Pt/C & 0.961 & 0.862
\end{tabular}

${ }^{a}$ The ORR onset potential is defined at which the current density reaches to $0.1 \mathrm{~mA} \mathrm{~cm}^{-2}$.

$\mathrm{C}-\mathrm{O}-\mathrm{C} / \mathrm{C}-\mathrm{N}$ in the $\mathrm{NC}$ to lower binding energies in comparison to those in the Co-CoO@NC/NC-800, and by Co 2p spectra, which shows the shifts of the peaks of $\mathrm{Co}^{(0)}$ and $\mathrm{Co}^{(\mathrm{II})}$ in the $\mathrm{Co}-$ CoO@NC/NC-800 to higher binding energies in comparison to those in the Co-CoO@C/C-800. Additionally, Fig. 3d also shows a difference between the $\mathrm{N} 1 \mathrm{~s}$ spectra profiles of the $\mathrm{NC}$ and the Co-CoO@NC/NC-800. This might be due to the fact that the growth of the $\mathrm{Co}-\mathrm{CoO}$ nanoparticles on the nitrogen doping sites makes some of the nitrogen atoms undetectable by the XPS spectroscopy due to the low penetrability of X-ray photoelectrons, or the presence of the Co precursor in the reaction mixture changes the relative percentages of the different nitrogen components in the Co-CoO@NC/NC-800.

Previous work have demonstrated that the presence of metal$\mathrm{N}$ bond in the metal and nitrogen coexisting system is responsible for the high catalytic activity for the ORR. ${ }^{54,55}$ In the work presented here, however, the metal-N bonds are not detected in both the XPS spectra of Co $2 \mathrm{p}$ and $\mathrm{N} 1 \mathrm{~s}$, which clearly rules out the presence of the metal-N bond in the Co-CoO@NC/NC-800 and its contribution to the higher catalytic activity of the CoCoO@NC/NC-800. On the basis of this and the results shown above, we therefore attribute the following aspects to the main reasons responsible for the high catalytic activity of the $\mathrm{Co}^{-}$ CoO@NC/NC-800: (1) strong electronic interaction between the $\mathrm{Co}-\mathrm{CoO}$ nanoparticles and the $\mathrm{NC},(2)$ high specific surface area of the Co-CoO@NC/NC-800, (3) small and uniformly sized Co$\mathrm{CoO}$ nanoparticles, (4) good electric conductivity of the CoCoO@NC/NC-800 due to its well carbonized structure, (5) good hydrophilicity of the Co-CoO@NC/NC-800 due to the presence of the NC. ${ }^{30,56}$ Additionally, the interaction between Co and $\mathrm{CoO}$ could be an additional reason leading to the high catalytic activity of the Co-CoO@NC/NC-800. As reported previously, the composites consisting of TMs and their oxides exhibit greatly enhanced catalytic activities in comparison to their single component counterparts. ${ }^{36,57,58}$

To gain insight into the reaction kinetics and mechanism of the ORR by the Co-CoO@NC/NC-800, its LSVs at the different rotating rates were recorded. Fig. 4 e shows an improvement of the measured current density with increase of the rotating rate. Analysis of the LSVs using the K-L equation shows a linear relationship between the inverse of the current density $\left(j^{-1}\right)$ and the inverse of the root of the rotating rate $\left(\omega^{-1 / 2}\right)$ at the different potentials (Fig. 4f). This indicates that the ORR by the CoCoO@NC/NC-800 follows a first order reaction kinetics with respect to the concentration of dissolved oxygen. According to the slopes of the K-L plots, the electron transfer number $(n)$ of the ORR can be extracted. Fig. $4 \mathrm{~g}$ shows that the electron transfer number of the ORR by the Co-CoO@NC/NC-800 is always higher than 3.8 and close to that of the $\mathrm{Pt} / \mathrm{C}$ over the potential range covered in this work, demonstrating that the ORR by the Co-CoO@NC/NC-800 proceeds mainly via a favorable four-electron pathway. This is different from the ORR by the NC, the Co-CoO@C/C-800, the Co-CoO@NC/NC-700, and Co-CoO@NC/NC-900 (the K-L plots of the NC, the Co-CoO@C/ C-800, the Co-CoO@NC/NC-700, and Co-CoO@NC/NC-900 are given in Fig. $\mathrm{S} 7 \dagger$ ), in which a two-electron pathway plays an increasing role in the reduction of oxygen. As shown in Fig. $4 \mathrm{~g}$, the electron transfer numbers of the ORR by the $\mathrm{NC}$, the Co$\mathrm{CoO} @ \mathrm{C} / \mathrm{C}-800$, the Co-CoO@NC/NC-700, and Co-CoO@NC/ NC-900 are much lower than 4.0. Fig. 4h presents the ORR kinetic current density $\left(j_{\mathrm{k}}\right)$ of the catalysts. It shows that the CoCoO@NC/NC-800 exhibits higher ORR kinetic current density than the NC, the Co-CoO@C/C-800, the Co-CoO@NC/NC-700, and Co-CoO@NC/NC-900, further demonstrating that the CoCoO@NC/NC-800 is a more efficient catalyst for the ORR than the NC, the Co-CoO@C/C-800, the Co-CoO@NC/NC-700, and Co-CoO@NC/NC-900.

The rotating ring-disk electrode (RRDE) measurement was perform to further investigate the ORR by the Co-CoO@NC/NC800. Fig. 5a shows the LSV of the ORR by the Co-CoO@NC/NC800 obtained from the disk electrode of the RRDE shows a profile similar to that directly obtained from the rotating disk electrode (Fig. 4b). Most interestingly, the ORR by the CoCoO@NC/NC-800 shows a comparable ring current corresponding to hydrogen peroxide ions $\left(\mathrm{H}_{2} \mathrm{O}^{-}\right)$to that by the $\mathrm{Pt} / \mathrm{C}$. Fig. 5b reveals that the $\mathrm{H}_{2} \mathrm{O}^{-}$yield of the ORR by the CoCoO@NC/NC-800 is below 5\% with an electron transfer number higher than 3.9 over the potential range from 0.2 to $0.8 \mathrm{~V}$. The $\mathrm{H}_{2} \mathrm{O}^{-}$yield and electron transfer number of the ORR by the CoCoO@NC/NC-800 are very close to those by the Pt/C, as shown Fig. 5b. Most interestingly, the $\mathrm{H}_{2} \mathrm{O}^{-}$yield of the ORR by the CoCoO@NC/NC-800 is lower than those of most catalysts reported previously, while its electron transfer number is higher than those of the ORR by most catalysts reported previously. ${ }^{36,59,60}$ This further demonstrates that the Co-CoO@NC/NC-800 has a catalytic activity close to that of the $\mathrm{Pt} / \mathrm{C}$.

In view that the Co-CoO@NC/NC-800 is highly active for the ORR with great potential for the replacement of the commercial $\mathrm{Pt} / \mathrm{C}$, its stability and durability towards the methanol crossover were tested. The current-time $(i-t)$ chronoamperometric curve in Fig. $5 \mathrm{c}$ shows that the current density of the ORR by the Pt/C decreases rapidly with increase of the operating time. This could be attributed to the low stability of the $\mathrm{Pt} / \mathrm{C}$ in the alkaline environment, which undergoes a fast degradation of the $\mathrm{Pt} / \mathrm{C}$ over the time due to the dissolution and aggregation of the $\mathrm{Pt}$ nanoparticles and their surface oxidation. ${ }^{\mathbf{6 1 , 6 2}}$ The introduction of methanol causes a sharp decrease in its ORR current density due to the poisoning of the ORR active sites by the oxidized products of methanol (Fig. 5d) ${ }^{62,63}$ The stability of the Co- 

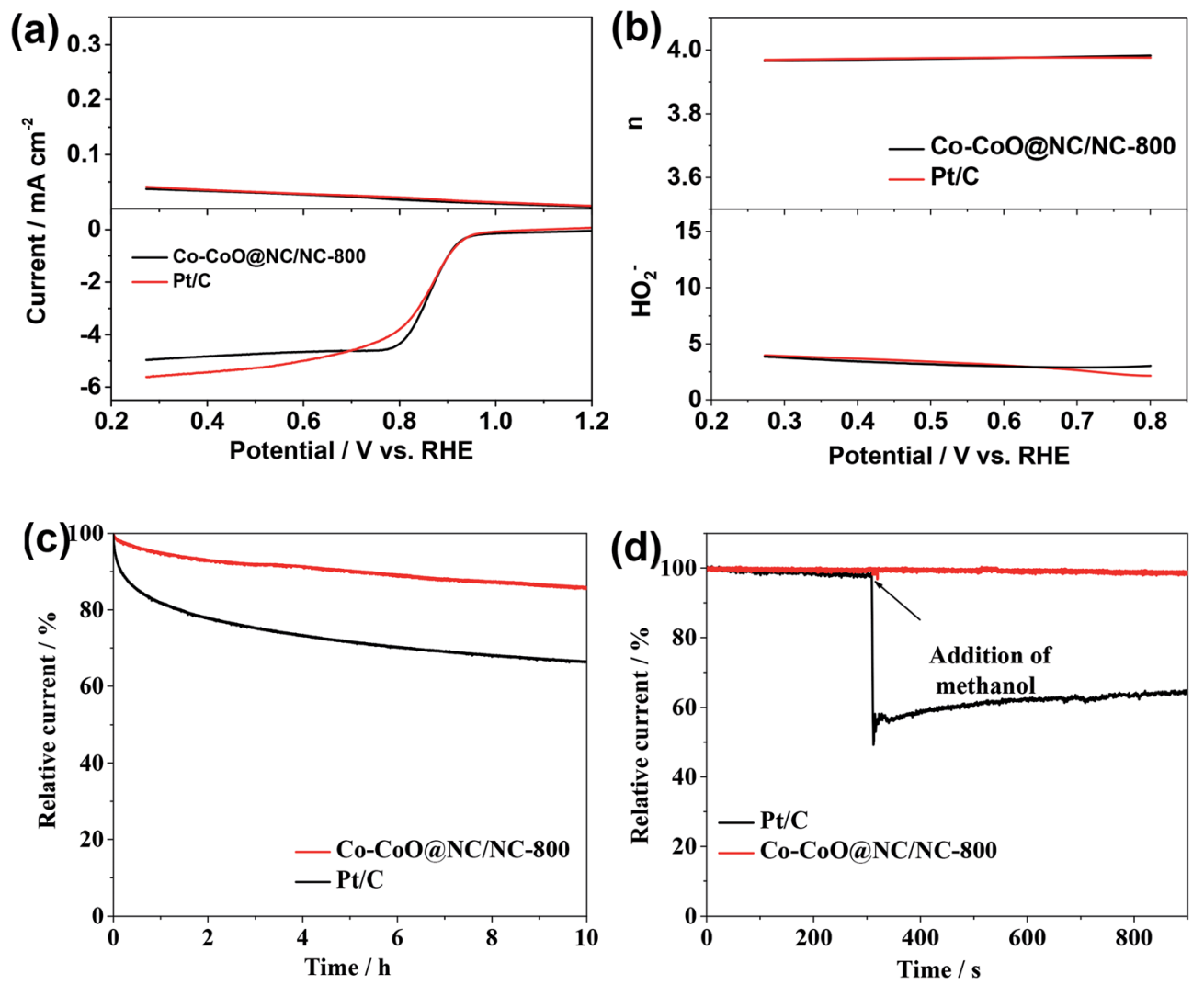

Fig. 5 (a) LSV curves of the Co-CoOaNC/NC-800 and the Pt/C obtained from the RRDE measurements at a rotation rate of $1600 \mathrm{rpm}$ in the $\mathrm{O}_{2}$ saturated $0.1 \mathrm{M} \mathrm{KOH}$ solution. (b) $\mathrm{HO}_{2}{ }^{-}$yields (top) and electron transfer number ( $n$, bottom) of the $\mathrm{Co}-\mathrm{CoO}$ aNC/NC-800 and the Pt/C obtained from the RRDE measurements. (c) Chronoamperometric curves of the Co-CoO@NC/NC-800 and the Pt/C at $0.67 \mathrm{~V}$ vs. RHE in the $\mathrm{O}_{2}-$ saturated $0.1 \mathrm{~mol} \mathrm{~L} \mathrm{~L}^{-1} \mathrm{KOH}$ solution. (d) Chronoamperometric curves of the Co-CoOaNC/NC-800 and the Pt/C electrodes with addition of $0.3 \mathrm{M}$ methanol. The arrow indicates the addition of methanol.

CoO@NC/NC-800 and its durability toward the methanol crossover are much higher than those of the Pt/C. As shown in Fig. $5 \mathrm{c}$ and $\mathrm{d}$, the current density of the ORR by the CoCoO@NC/NC-800 decreases much slowly in comparison to that by the $\mathrm{Pt} / \mathrm{C}$, and the introduction of methanol has a negligible effect on its ORR current density. These results strongly suggest that the specific coating structure of the $\mathrm{Co}-\mathrm{CoO}$ nanoparticles by the NC shell in the Co-CoO@NC/NC-800 could effectively protect the $\mathrm{Co}-\mathrm{CoO}$ nanoparticles from the degradation and poisoning of the ORR active sites by the oxidized products of methanol.

\section{Conclusions}

In summary, the $\mathrm{Co}-\mathrm{CoO} @ \mathrm{NC} / \mathrm{NC}-800$, which consists of the $\mathrm{NC}$ nanosheets with deposition of the NC coated $\mathrm{Co}-\mathrm{CoO}$ nanoparticles, has been synthesized through a simple calcination of the dried reaction mixture of $\mathrm{Co}\left(\mathrm{NO}_{3}\right)_{2}$, glucose, and urea. The Co-CoO@NC/NC-800 is highly active for the ORR and can deliver an onset potential of $0.961 \mathrm{~V} v s$. RHE and a half-wave potential of $0.868 \mathrm{~V}$ vs. RHE. The onset and the half-wave potentials of the $\mathrm{Co}-\mathrm{CoO} @ \mathrm{NC} / \mathrm{NC}-800$ are very close to those of the $\mathrm{Pt} / \mathrm{C}$. This, in conjunction with its higher stability and better durability towards the methanol crossover, strongly suggests that the $\mathrm{Co}-\mathrm{CoO} @ \mathrm{NC} / \mathrm{NC}-800$ is a promising ORR catalyst for practical uses. The work presented here does not simply introduce a new and simple method for the synthesis of the ORR catalysts of high efficiency, and the same method might be also applicable to design and synthesize other catalysts for various applications.

\section{Conflicts of interest}

There are no conflicts to declare.

\section{Acknowledgements}

This work is financially supported from the Chinese National Natural Science Foundation (No. 11474101 and U1532139), the "Outstanding Talent and Team Plans Program" of South China University of Technology, the Guangdong Provincial Natural Science Foundation (No. 2017A030313092), the Guangdong Innovative and Entrepreneurial Research Team Program (No. 2014ZT05N200), and the Ningbo Natural Science Foundation (No. 2017A610059).

\section{References}

1 S. K. Singh, V. M. Dhavale and S. Kurungot, ACS Appl. Mater. Interfaces, 2015, 7, 21138-21149. 
2 L. Li, C. Liu, G. He, D. Fan and A. Manthiram, Energy Environ. Sci., 2015, 8, 3274-3282.

3 K. Mohanraju and L. Cindrella, RSC Adv., 2014, 4, 1193911947.

4 Y. Jiao, Y. Zheng, M. Jaroniec and S. Z. Qiao, Chem. Soc. Rev., 2015, 44, 2060-2086.

5 L. Bu, S. Guo, X. Zhang, X. Shen, D. Su, G. Lu, X. Zhu, J. Yao, J. Guo and X. Huang, Nat. Commun., 2016, 7, 11850-11859.

6 G. Hu, E. Gracia-Espino, R. Sandström, T. Sharifi, S. Cheng, H. Shen, C. Wang, S. Guo, G. Yang and T. Wågberg, Catal. Sci. Technol., 2016, 6, 1393-1401.

7 J. Ding, X. Zhu, L. Bu, J. Yao, J. Guo, S. Guo and X. Huang, Chem. Commun., 2015, 51, 9722-9725.

8 Y. Zhao, J. Liu, Y. Zhao and F. Wang, Phys. Chem. Chem. Phys., 2014, 16, 19298-19306.

9 D. U. Lee, M. G. Park, H. W. Park, M. H. Seo, X. Wang and Z. Chen, ChemSusChem, 2015, 8, 3129-3138.

10 P. Sennu, M. Christy, V. Aravindan, Y.-G. Lee, K. S. Nahm and Y.-S. Lee, Chem. Mater., 2015, 27, 5726-5735.

11 C. Zhu, H. Li, S. Fu, D. Du and Y. Lin, Chem. Soc. Rev., 2016, 45, 517-531.

12 H. J. Choi, N. A. Kumar and J. B. Baek, Nanoscale, 2015, 7, 6991-6998.

13 J. E. Kim, J. Lim, G. Y. Lee, S. H. Choi, U. N. Maiti, W. J. Lee, H. J. Lee and S. O. Kim, ACS Appl. Mater. Interfaces, 2016, 8, 1571-1577.

14 M. Shen, C. Ruan, Y. Chen, C. Jiang, K. Ai and L. Lu, ACS Appl. Mater. Interfaces, 2015, 7, 1207-1218.

15 Y. Zhan, M. Lu, S. Yang, C. Xu, Z. Liu and J. Y. Lee, ChemCatChem, 2016, 8, 372-379.

16 J. Masa, W. Xia, I. Sinev, A. Zhao, Z. Sun, S. Grutzke, P. Weide, M. Muhler and W. Schuhmann, Angew. Chem., Int. Ed., 2014, 53, 8508-8512.

17 M. Sun, H. Liu, Y. Liu, J. Qu and J. Li, Nanoscale, 2015, 7, 1250-1269.

18 J. Xu, Q. Yu, C. Wu and L. Guan, J. Mater. Chem. A, 2015, 3, 21647-21654.

19 S. Guo, S. Zhang, L. Wu and S. Sun, Angew. Chem., Int. Ed., 2012, 51, 11770-11773.

20 H. Yu, Y. Li, X. Li, L. Fan and S. Yang, Chem.-Eur. J., 2014, 20, 3457-3462.

21 M. Li, X. Bo, Y. Zhang, C. Han, A. Nsabimana and L. Guo, J. Mater. Chem. A, 2014, 2, 11672-11682.

22 Z. Wang, S. Xiao, Z. Zhu, X. Long, X. Zheng, X. Lu and S. Yang, ACS Appl. Mater. Interfaces, 2015, 7, 4048-4055.

23 Y. Li, F. Cheng, J. Zhang, Z. Chen, Q. Xu and S. Guo, Small, 2016, 12, 2839-2845.

24 J. Deng, P. Ren, D. Deng and X. Bao, Angew. Chem., 2015, 127, 2128-2132.

25 D. Deng, L. Yu, X. Chen, G. Wang, L. Jin, X. Pan, J. Deng, G. Sun and X. Bao, Angew. Chem., Int. Ed., 2013, 52, 371-375.

26 Y. Wang, X. Ding, F. Wang, J. Li, S. Song and H. Zhang, Chem. Sci., 2016, 7, 4284-4290.

27 M. Zhuang, X. Ou, Y. Dou, L. Zhang, Q. Zhang, R. Wu, Y. Ding, M. Shao and Z. Luo, Nano Lett., 2016, 16, 4691-4698.

28 T. Kottakkat and M. Bron, ChemElectroChem, 2014, 1, 21632171.
29 K. Kreek, A. Sarapuu, L. Samolberg, U. Joost, V. Mikli, M. Koel and K. Tammeveski, ChemElectroChem, 2015, 2, 2079-2088.

30 C. Han, X. Bo, Y. Zhang, M. Li, A. Nsabimana and L. Guo, Nanoscale, 2015, 7, 5607-5611.

31 X. Zhang, R. Liu, Y. Zang, G. Liu, G. Wang, Y. Zhang, H. Zhang and H. Zhao, Chem. Commun., 2016, 52, 59465949.

32 H. Zhong, Y. Luo, S. He, P. Tang, D. Li, N. Alonso-Vante and Y. Feng, ACS Appl. Mater. Interfaces, 2017, 9, 2541-2549.

33 K. Liu, X. Huang, H. Wang, F. Li, Y. Tang, J. Li and M. Shao, ACS Appl. Mater. Interfaces, 2016, 8, 34422-34430.

34 A. B. Yousaf, M. Imran, N. Uwitonze, A. Zeb, S. J. Zaidi, T. M. Ansari, G. Yasmeen and S. Manzoor, J. Phys. Chem. C, 2017, 121, 2069-2079.

35 Y. Cheng, S. Dou, M. Saunders, J. Zhang, J. Pan, S. Wang and S. P. Jiang, J. Mater. Chem. A, 2016, 4, 13881-13889.

36 Z. Wu, J. Wang, L. Han, R. Lin, H. Liu, H. L. Xin and D. Wang, Nanoscale, 2016, 8, 4681-4687.

37 T.-Y. Yung, L.-Y. Huang, T.-Y. Chan, K.-S. Wang, T.-Y. Liu, P.-T. Chen, C.-Y. Chao and L.-K. Liu, Nanoscale Res. Lett., 2014, 9, 444.

38 X. Dong, H. Xu, X. Wang and Y. Huang, ACS Nano, 2012, 6, 3206-3213.

39 Z. J. Jiang and Z. Jiang, Sci. Rep., 2016, 6, 27081.

40 S. Wang, J. Zhang, P. Shang, Y. Li, Z. Chen and Q. Xu, Chem. Commun., 2014, 50, 12091-12094.

41 Z. Lin, G. Waller, Y. Liu, M. Liu and C.-P. Wong, Adv. Energy Mater., 2012, 2, 884-888.

42 C. Ma, X. Shao and D. Cao, J. Mater. Chem. A, 2012, 22, 89118915.

43 Y.-X. Yu, Phys. Chem. Chem. Phys., 2013, 16819-16827.

44 C. V. Rao, C. R. Cabrera and Y. Ishikawa, J. Phys. Chem. Lett., 2010, 1, 2622-2627.

45 S. Yang, X. Feng, X. Wang and K. Müllen, Angew. Chem., Int. Ed., 2011, 50, 5339-5343.

46 X. Zhang, D. Yu, Y. Zhang, W. Guo, X. Ma and X. He, RSC Adv., 2016, 6, 104183-104192.

47 S. Cao, H. Chen, F. Jiang and X. Wang, Appl. Catal., B, 2018, 224, 222-229.

48 Y. Liang, H. Wang, J. Zhou, Y. Li, J. Wang, T. Regier and H. Dai, J. Am. Chem. Soc., 2012, 134, 3517-3523.

49 Y. Liang, H. Wang, P. Diao, W. Chang, G. Hong, Y. Li, M. Gong, L. Xie, J. Zhou, J. Wang, T. Z. Regier, F. Wei and H. Dai, J. Am. Chem. Soc., 2012, 134, 15849-15857.

50 D. Gao, R. Shibuya, C. Akiba, S. Saji, T. Kondo and J. Nakamura, Science, 2016, 351, 361-365.

51 L. Lai, J. R. Potts, D. Zhan, L. Wang, C. K. Poh, C. Tang, H. Gong, Z. Shen, J. Lin and R. S. Ruoff, Energy Environ. Sci., 2012, 5, 7936-7942.

52 Z. Jiang, Z.-j. Jiang, X. Tian and W. Chen, J. Mater. Chem. A, 2014, 2, 441-450.

53 Z.-J. Jiang and Z. Jiang, J. Mater. Chem. A, 2014, 2, 1407114081.

54 C. Li, Z. Han, Y. Yu, Y. Zhang, B. Dong, A. Kong and Y. Shan, RSC Adv., 2016, 6, 15167-15174. 
55 Y. Jiang, Y. Lu, X. Wang, Y. Bao, W. Chen and L. Niu, Nanoscale, 2014, 6, 15066-15072.

56 D.-W. Wang and D. Su, Energy Environ. Sci., 2014, 7, 576-591. 57 V. M. Dhavale, S. K. Singh, A. Nadeem, S. S. Gaikwad and S. Kurungot, Nanoscale, 2015, 7, 20117-20125.

58 X. X. Liu, J. B. Zang, L. Chen, L. B. Chen, X. Chen, P. Wu, S. Y. Zhou and Y. H. Wang, J. Mater. Chem. A, 2017, 5, 5865-5872.
59 C. Han, X. Bo, Y. Zhang, M. Li, A. Wang and L. Guo, Chem. Commun., 2015, 51, 15015-15018.

60 G. L. Tian, M. Q. Zhao, D. Yu, X. Y. Kong, J. Q. Huang, Q. Zhang and F. Wei, Small, 2014, 10, 2251-2259.

61 Y. Nie, L. Li and Z. Wei, Chem. Soc. Rev., 2015, 44, 2168-2201.

62 H. Uchida, K. Izumi and M. Watanabe, J. Phys. Chem. B, 2006, 110, 21924-21930.

63 F. Tian and A. B. Anderson, J. Phys. Chem. C, 2008, 112, 18566-18571. 